\title{
Advances on silicon-based integrated microwave photonics
}

Dong, Jianji; Yan, Siqi; Wang, Xu; Qiu, Huaqing; Zhao, Yuhe; Yu, Yuan; Ding, Yunhong; Xiao, Sanshui ; Zhang, Xinliang

\section{Published in:}

Proceedings of Spie

Link to article, DOI:

$10.1117 / 12.2286443$

Publication date:

2018

Document Version

Publisher's PDF, also known as Version of record

Link back to DTU Orbit

Citation (APA):

Dong, J., Yan, S., Wang, X., Qiu, H., Zhao, Y., Yu, Y., Ding, Y., Xiao, S., \& Zhang, X. (2018). Advances on silicon-based integrated microwave photonics. In Proceedings of Spie (Vol. 10536). [105361 C] SPIE International Society for Optical Engineering. Proceedings of SPIE - The International Society for Optical Engineering https://doi.org/10.1117/12.2286443

\section{General rights}

Copyright and moral rights for the publications made accessible in the public portal are retained by the authors and/or other copyright owners and it is a condition of accessing publications that users recognise and abide by the legal requirements associated with these rights.

- Users may download and print one copy of any publication from the public portal for the purpose of private study or research.

- You may not further distribute the material or use it for any profit-making activity or commercial gain

- You may freely distribute the URL identifying the publication in the public portal 


\section{Advances on silicon-based integrated microwave photonics}

Jianji Dong, Siqi Yan, Xu Wang, Huaqing Qiu, Yuhe Zhao, et al.

Jianji Dong, Siqi Yan, Xu Wang, Huaqing Qiu, Yuhe Zhao, Yuan Yu, Yunhong Ding, Sanshui Xiao, Xinliang Zhang, "Advances on silicon-based integrated microwave photonics," Proc. SPIE 10536, Smart Photonic and Optoelectronic Integrated Circuits XX, 105361C (22 February 2018); doi: 10.1117/12.2286443

SPIE. Event: SPIE OPTO, 2018, San Francisco, California, United States 


\title{
Advances on silicon-based integrated microwave photonics
}

\author{
Jianji Dong*a, Siqi Yan ${ }^{\mathrm{a}}$, Xu Wang ${ }^{\mathrm{a}}$, Huaqing Qiu ${ }^{\mathrm{a}}$, Yuhe Zhao ${ }^{\mathrm{a}}$, Yuan $\mathrm{Yu}^{\mathrm{a}}$, Yunhong Ding ${ }^{* * \mathrm{~b}}$, \\ Sanshui Xiao ${ }^{b}$, and Xinliang Zhang ${ }^{\text {a }}$ \\ ${ }^{a}$ Wuhan National Laboratory for Optoelectronics, Huazhong University of Science and Technology, \\ Wuhan 430074, China; ${ }^{\mathrm{b}}$ Department of Photonics Engineering Denmark Technical University, \\ Copenhagen, Denmark
}

\begin{abstract}
Microwave photonic systems have huge potential for both existing and future applications, including radar, radiofrequency sensing and modern wireless communications due to their distinct advantages in terms of ultra-wide bandwidth, flexible tunability, and immunity to electromagnetic interference. There is a strong research trend in microwave photonic systems towards integration and miniaturization, resulting in multiple radio frequency functions on a single chip which is both compact and light weight. Thus integrated microwave photonics has attracted a lot of attentions and achieves significant improvements in last ten years. In this paper, we will review some research progresses on silicon-based integrated microwave photonics in our group, including highly efficient micro heater on silicon photonic chip, chip-scale microwave waveform generation, on-chip true time delay, and microwave photonic processing and measurement. Our schemes are all fabricated on silicon-on-insulator chips and have advantages of compactness and capability to integrate with electronic units. These chips may motivate the great application potentials in silicon-based integrated microwave photonics.
\end{abstract}

Keywords: Microwave photonics, Silicon photonics, Microwave signal processing

\section{INTRODUCTION}

Microwave photonic systems have huge potential for both existing and future applications, including radar, radio frequency (RF) sensing and modern wireless communications such as existing Long-Term Evolution (LTE) technology and upcoming the fifth generation $(5 \mathrm{G})$ networks. This is because they have distinct advantages in terms of ultra-wide bandwidth, flexible tunability, and immunity to electromagnetic interference. The applications of microwave photonics cover civil use and military project, such as electronic countermeasure, radar system [1], wireless communications [2], sensing, optical ultra-wide band (UWB) signal generation [3, 4], all photonic temporal differentiator [5, 6], and test of optical communication system. The trend in microwave photonic systems research is towards integration and chip miniaturization, which will result in advanced multi-component chips with greatly reduced size and weight compared to the conventional discrete component approach.

In the past decades, there were a lot of outstanding advances on integrated microwave photonics. Thanks to the mature silicon CMOS foundry, excellent schemes for radio-frequency arbitrary waveform generation (AWG) were demonstrated [7-11]. Especially, the reconfigurable AWG scheme can generate programmable radio-frequency bursts in a silicon photonic chip which can modulate the pulse burst within 4 ns [7]. Besides, Mahmoud S. Rasras et al has demonstrated a wide tuning-range optical delay line which is based on silica planar light wave circuits [12]. The time delay can vary from 0 to $2.56 \mathrm{~ns}$ and the propagation loss is only $0.07 \mathrm{~dB} / \mathrm{cm}$. Recently, a reconfigurable mesh network on a chip was demonstrated to enable multifunction of microwave processor, such as true time delay (TTD), microwave photonic filter (MPF), and phase arrayed antenna (PAA) [13]. And a monolithic integrated photonic microwave filter is proposed by José Capmany [14]. It is the first time that the lasers, modulators and photodetectors are all integrated into a single indium phosphide chip. In terms of microwave frequency measurement (MFM), Eggleton et al has demonstrated excellent MFM system based on stimulated Brillouin scattering in chalcogenide glass waveguide [15-17]. However, those chip-scale microwave photonic systems still face several technical challenges and difficulties including the poor frequency-tuning accuracy, high electrical power consumption and slow tuning speed.

*jidong@ hust.edu.cn; **yudin@fotonik.dtu.dk

Smart Photonic and Optoelectronic Integrated Circuits XX, edited by Sailing $\mathrm{He}$,

El-Hang Lee, Proc. of SPIE Vol. 10536, 105361C · C) 2018 SPIE

CCC code: $0277-786 \mathrm{X} / 18 / \$ 18 \cdot$ doi: $10.1117 / 12.2286443$ 
In this paper, we will review some representative research progresses on integrated microwave photonics in our group, including highly efficient microheater on silicon photonic chip, chip scale microwave waveform generation, on-chip true time delay, and microwave photonic processing and measurement. First, we will demonstrate an energy-efficient graphene microheater with a tuning efficiency of $1.07 \mathrm{~nm} / \mathrm{mW}$ and power consumption per free spectral range (FSR) of $3.99 \mathrm{~mW}$. The rise and decay times (10\% to 90\%) are only $750 \mathrm{~ns}$ and $525 \mathrm{~ns}$. Second, we will demonstrate several typical schemes about microwave arbitrary waveform generation including the finite impulse response synthesis and Tylor synthesis. Third, we will demonstrate an on-chip TTD unit based on chirped grating waveguide. Finally, we will demonstrate some microwave processors, such as MFM and microwave photonic filter (MPF). Our schemes are all fabricated on silicon-on-insulator (SOI) chips and have advantages of compactness and capability to integrate with electronic devices. These chips may have great application potentials in silicon-based integrated microwave photonics.

\section{NOVEL THERMAL TUNING SCHEME IN SILICON PHOTONICS}

The thermal tuning acts as one of the most crucial functionalities in microwave photonics [18]. The flexibility and tunability of the advanced microwave photonics are very dependent on the thermal tuning in basic units including optical switches, phase shifters as well as the intensity modulators. To realize both low power consumption and fast tuning remains to be a major issue since the traditional metallic microheater requires a thick silicon dioxide $\left(\mathrm{SiO}_{2}\right)$ layer introduced between the silicon waveguide and the metallic heater to avoid the light-absorption loss, thus inevitably impeding heat transport and dissipation owing to the low thermal conductivity of $\mathrm{SiO}_{2}$. Several schemes have been proposed to reduce the tuning power and response time, including the free-standing waveguide [19,20], different doping levels [21] of the waveguide, and graphene microheater [22, 23]. However, the response time of free-standing waveguide remains slow while the tuning efficiency of different silicon waveguide doping is low [21]. Here we have proposed two different methods to realize the efficient thermal tuning in integrated silicon photonics. The first scheme is the slowlight-enhanced graphene heater [23] and the second scheme is based on the side-integrated metallic heater with photonic crystal waveguide $(\mathrm{PhCW})$.

The structure of the slow light enhanced graphene heater is shown in Fig. 1(a). A graphene monolayer is deposited onto the core-region of the silicon $\mathrm{PhCW}$ to act as the heater. The width of the graphene overlapping the photonic crystal line defect is designed to be narrower than the other part of the graphene to locally increase the Ohmic dissipation, which results in a more effective heating. According to the experiment results, the slow light enhanced tuning efficiency is 1.07 $\mathrm{nm} / \mathrm{mW}$, as Fig. 1(b) displays. In addition, the response time can be as fast as $525 \mathrm{~ns}$, as the Fig. 1(c-d) shows. This is the fastest reported response times for microheaters in silicon photonics, to the best of our knowledge. The corresponding figure of merit of the device is $2.543 \mathrm{nW} \cdot \mathrm{s}$, one order of magnitude better than results reported in previous studies.
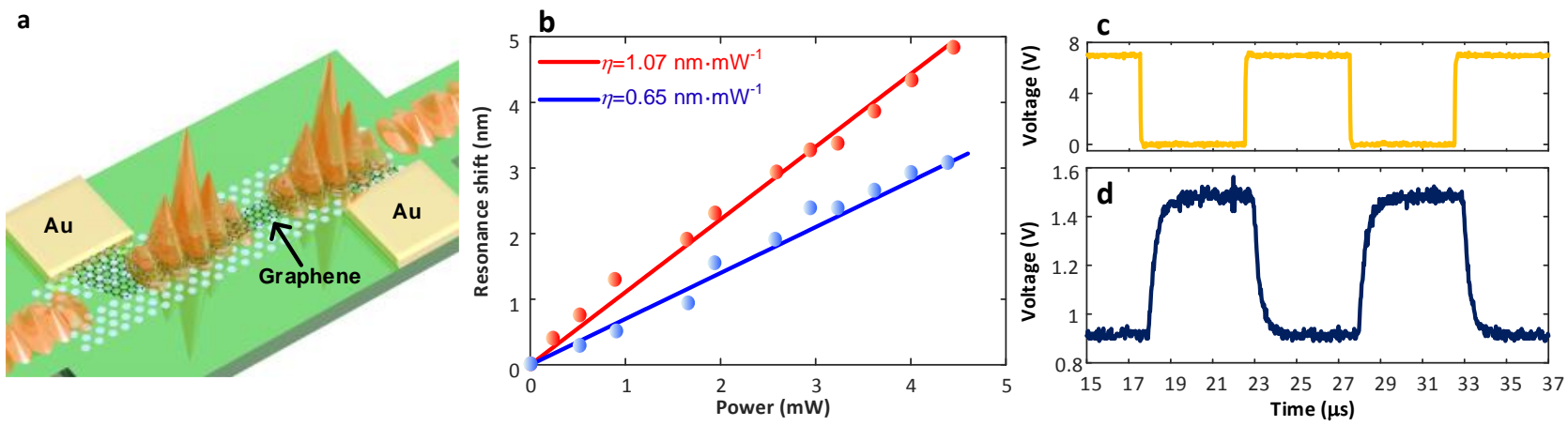

Figure 1. (a) The schematic of the slow-light-enhanced graphene microheater. (b) Measured resonance shifts for the interference dips with (red) and without (blue) the slow light effect as functions of the applied heating power. (c) Driving electrical signal and (d) corresponding temporal response signal.

Although graphene heater can provide the ultrafast response and low power consumption, the performance of the graphene heater faces several challenges including the excess loss induced by the fabrication of graphene heater, as well as the quality of graphene. To avert these difficulties, we propose the side-integrated metallic heater with the slow light effect, which is fully compatible with the conventional Complementary Metal-Oxide-Semiconductor (CMOS) fabrication process with fast response time and slow light enhanced tuning efficiency. The schematic of the proposed structure is shown in Fig. 2(a). To enable the tunability of the unit, the $\mathrm{NiCr}$ microheater is fabricated on the side of 
$\mathrm{PhCW}$, rather on the top of the waveguide. Therefore, the thick oxide layer in the conventional heating scheme can be prevented. The tuning efficiency with the slow light effect is $0.215 \mathrm{~nm} / \mathrm{mW}$ while the tuning efficiency without the slow light effect is $0.132 \mathrm{~nm} / \mathrm{mW}$, as the Fig. 2(b) shows. The response time of the proposed structure is $2 \mu \mathrm{s}$ according to the experimental results in Fig. 2(d).

a

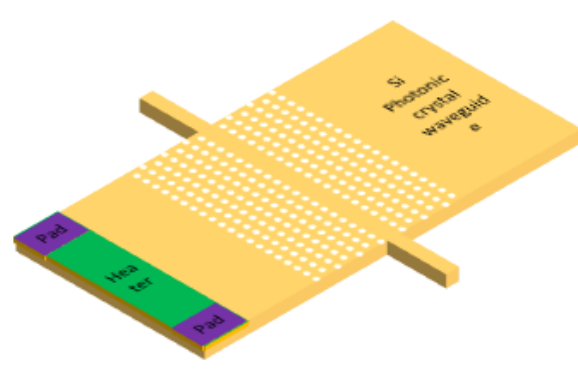

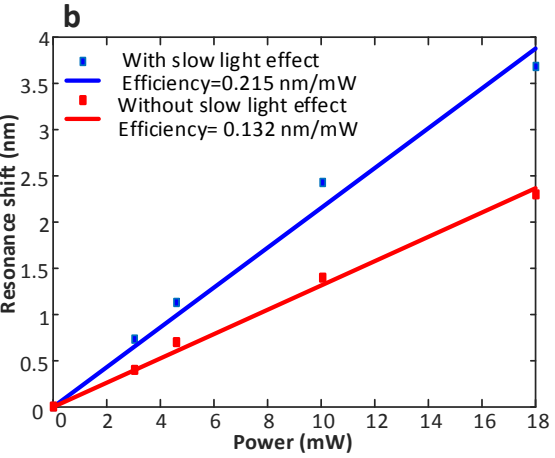

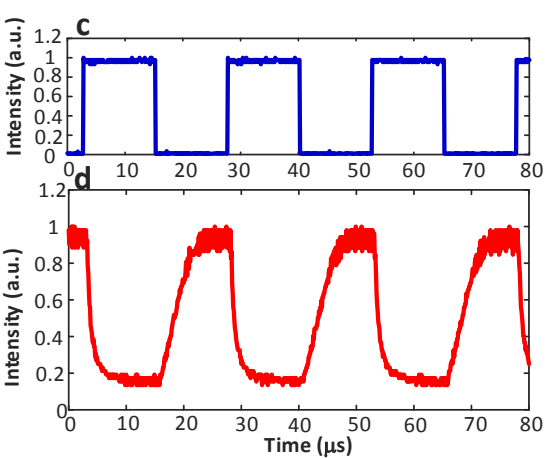

Figure 2. (a) The schematic of the side-integrated metal microheater with slow light effect. (b) Measured resonance shifts for the interference dips with (blue) and without (red) the slow light effect as functions of the applied heating power. (c) Driving electrical signal and (d) corresponding temporal response signal.

\section{ON-CHIP ARBITRARY WAVEFORM GENERATOR}

a

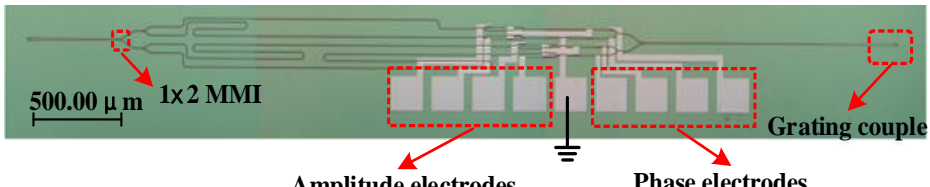

b
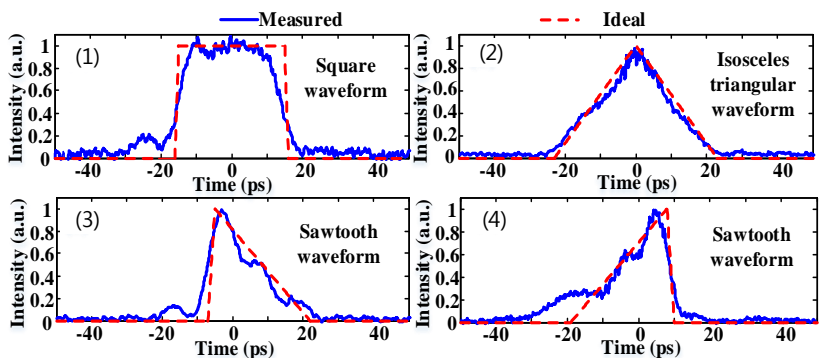

d
C

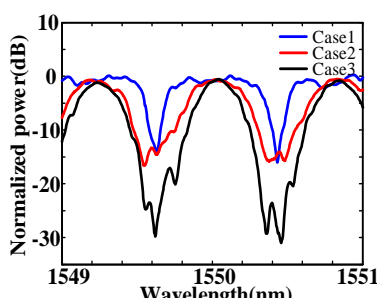

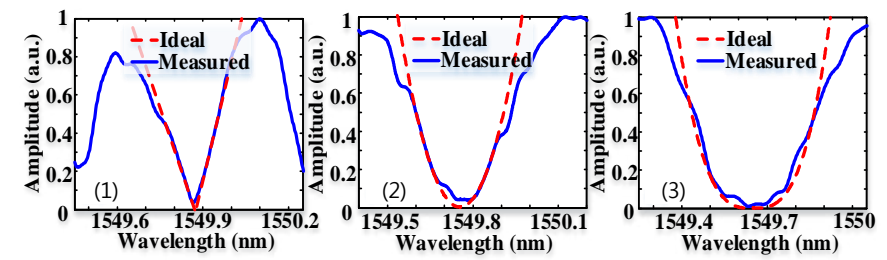

Figure 3. (a)Schematic diagram of the FIR on-chip optical arbitrary waveform generator. (b) Measured waveforms (blue solid line) of the pulse shaper and ideal ones (red dash line) of (1) square waveform, (2) isosceles triangular waveform, (3) and (4) sawtooth waveform. (c) measured spectra of the filter with bandwidth tunability. (d)Measured and ideal spectra of (1) first-, (2) second-, and (3) third-order differentiators.

Optical arbitrary waveform generation (OAWG) is widely applied in various fields, such as high speed optical communications [24], ultra-wideband signals [2], radar systems [25], all-optical signal processing including optical differentiator [26] and optical integrator [27, 28]. Besides, the photonic-based high frequency microwave signal 
generation also shows great importance in application, since it is superior to the traditional methods in many aspects such as speed, bandwidth and power consumption, thanks to the absence of electronic bottleneck. We have demonstrated several approaches for OWAG and microwave signal generation.

One approach is based on a four-tap finite impulse response (FIR) SOI chip [29], as shown in Fig. 3(a). Cascaded multimode interferometers are used to divide the signal into four taps. Within each tap the transfer function of the system can be adjusted thermally in the amplitude and the phase, consequently reshaping the output spectrum, which ensures the capability of generating various waveforms, shown in Fig. 3(b). Fig. 3(c) shows that, due to the tunability, the device can also serve as a programmable photonic filter. This device can also act as first-, second- and third-order differentiators [30], as shown in Fig. 3(d).

Besides, we have investigated the FIR method and applied it to 125-GHz microwave waveform generation. The scheme contains eight taps of FIR fabricated on a silicon chip, and each of taps has an amplitude modulator which can be thermally tuned [31]. Fig. 4(a) shows the schematic diagram of this scheme. When amplitude modulators, controlled by Mach-Zehnder interferometer (MZI), are particularly tuned, typical types of microwave waveforms such as square waveform, isosceles triangular waveform, trapezoid waveform, sawtooth waveform, are generated, as shown in Fig. 4(b).

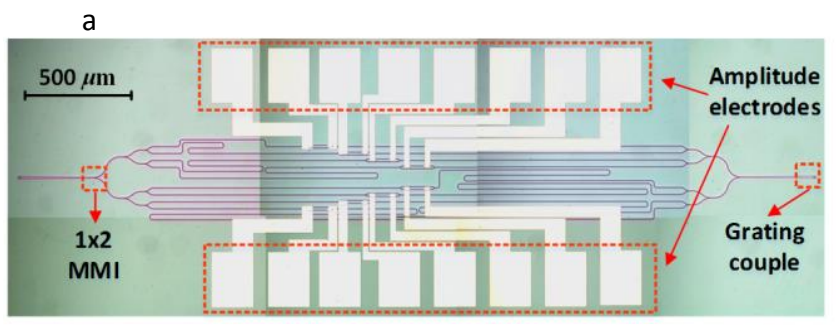

b

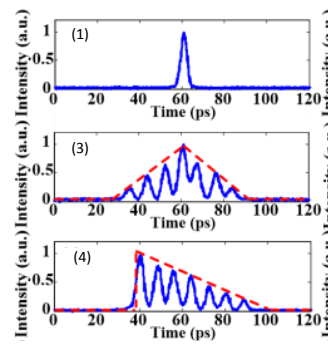

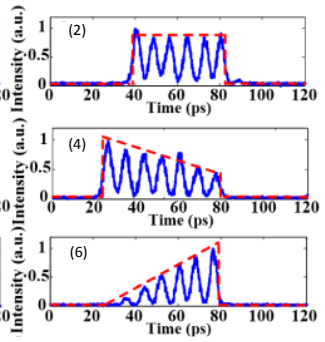

Figure 4. (a) Metallurgical microscopy image of the on-chip pulse shaper. (b) (1) Input pulse, measured waveforms (blue solid line) and ideal microwave waveforms (red dash line) of (2) $125 \mathrm{GHz}$ pulse burst with square envelope, (3) $125 \mathrm{GHz}$ pulse burst with isosceles triangular envelope, (4) $125 \mathrm{GHz}$ pulse burst with trapezoid envelope, (5) and (6) $125 \mathrm{GHz}$ pulse bursts with sawtooth envelope.
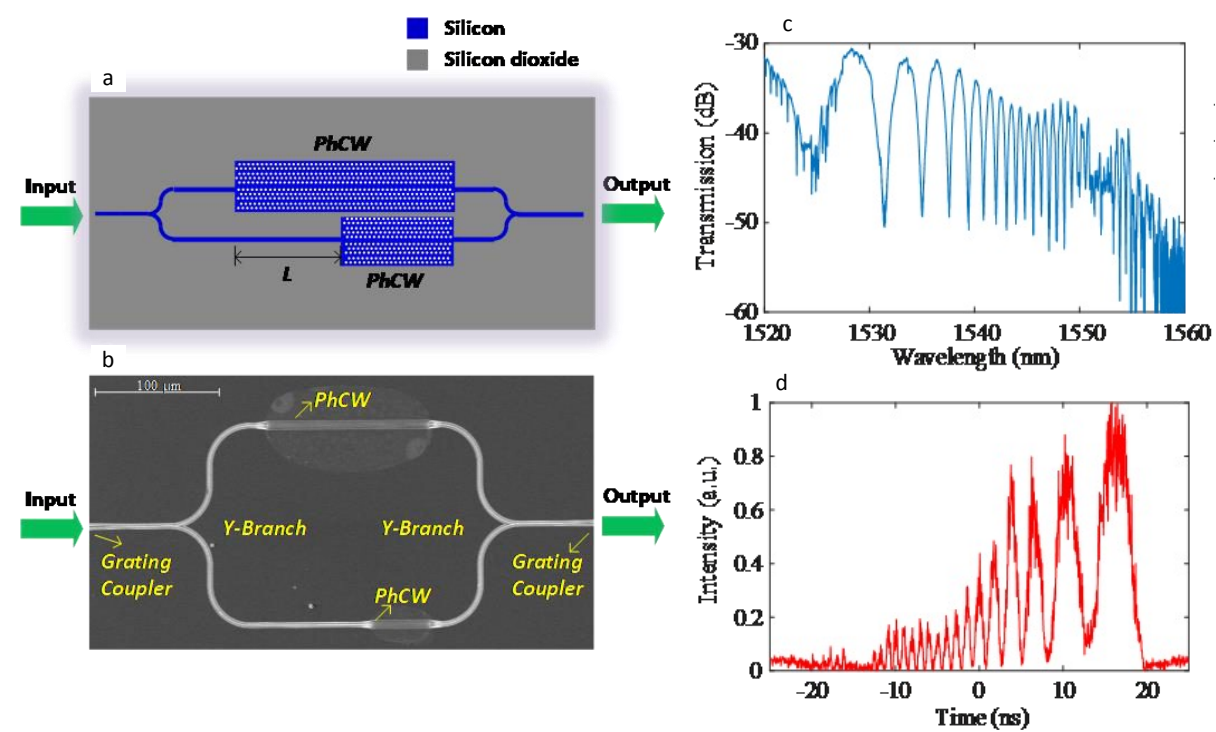

Figure 5. (a) The schematic of the PhCW-MZI. (b) The Scanning Electron Microscope (SEM) image of the PhCW-MZI. (c) The measured transmission spectrum of the PhCW-MZI. (d) The temporal waveform of the linear chirp microwave signal.

We have also presented the linearly chirped microwave pulse generation based on slow light effect within a photonic crystal waveguide [8] in Fig. 5(a). In this design, the two arms of MZI consist of two different lengths of PhCW, indicating different group velocity in each arm. The Scanning Electron Microscope (SEM) image of the PhCW-MZI is shown in Fig. 5(b). Therefore, the transfer function of the PhCW-MZI has a frequency-dependent free spectrum range response. Then a dispersive element is applied, that can realize frequency to time mapping to generate linearly chirped 
microwave signal. As is illustrated in experiments, our design has a negative chirp rate of $0.045 \mathrm{GHz} / \mathrm{ns}$ with the timebandwidth product (TBWP) of 30. Figs. 5(c) and (d) show the measured transmission spectrum of the PhCW-MZI and the temporal waveform of the linearly chirp microwave signal, respectively. Besides, the footprint of the proposed chip is only $0.096 \mathrm{~mm}^{2}$, which is one-order smaller than previous on-chip linearly chirped microwave generation schemes.

\section{TRUE TIME DELAY BASED ON SILICON PHOTONICS}

True time delay has a very wide range of applications in both optical and microwave fields, such as optical dispersion compensation [32], optically controlled phased array radar [33] and photonic generation of microwave waveforms [34]. In the recent years, on-chip optical delay lines have attracted great attentions due to the mature CMOS process. An intuitive solution is based on optical switch, which can realize tunable delay by changing the state of switches connected with waveguides in different lengths [35]. This method can realize large delay, while the tunable accuracy is limited by the shortest distance between switches and the dimensions are usually centimeter-scale. In addition, the scheme based on photonic crystal waveguides and integrated microring have been employed for the compact structure and large delay, while they are troubled by high insertion loss or limited operating bandwidth [36-38]. At the same time, combing Bragg gratings and thermo-optical effect, continuously tunable delay line based on SOI tapered Bragg gratings have been proposed and verified experimentally [39, 40]. Chirped waveguide Bragg gratings have low insertion loss, good reconfigurable ability and broad bandwidth, which is promising in future optical systems.
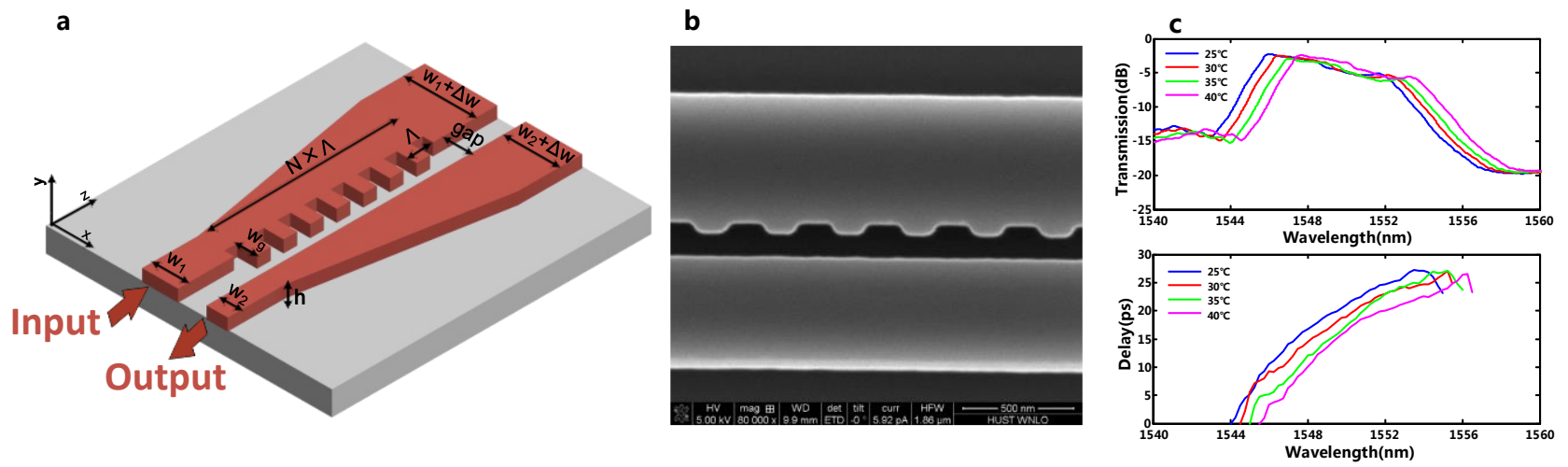

Figure 6. (a) The schematic of the contra-directional couplers with single sidewall-modulated Bragg gratings. (b) The SEM images of the contra-directional couplers (c) Reflection spectra and group delay of the contra-directional couplers at different temperatures.

As shown in Fig. 6(a), the proposed design is based on tapered SOI contra-directional couplers with single sidewallmodulated Bragg gratings [41]. The contra-directional couplers consist of two tapered rib waveguides with different width, and the Bragg gratings are modulated in the inner sidewall of the wider one. The gratings period keeps the same along the propagation direction and tapered waveguides are designed for the index chirping. The optical signal is launched from the wide waveguide and coupled to the narrow waveguide through the Bragg gratings structure. Along the direction of light propagation, the waveguide width varies linearly, so the reflection wavelength is different at different positions. As the device is based on stripe waveguide, it can be fabricated by one-step etching and the manufacturing process is simplified. Fig. 6(b) shows the SEM image of the fabricated contra-directional couplers with single sidewallmodulated Bragg gratings. In the experiment, the group delay response can be calculated by comparing the time difference of waveforms with different wavelength. As shown in Fig. 6(c), group delay of $28 \mathrm{ps}$ is realized over the passband of $10 \mathrm{~nm}$. By controlling the operating temperature with a Thermoelectric Cooler (TEC) ceramic chip, the delay can be continuously tuned employing the thermo-optical effect.

To increase the group delay time, we need to increase the total length of the gratings. For the aim of further integration, the on-chip thermal electrodes become the next step to be considered. However, the heating zone consists of the whole gratings, and the length of the gratings is quite long for the ordinary thermal electrodes. Thus, parallel electrodes design, which is shown in Fig. 7(a), is considered to reduce the overall resistance and realize larger spectral drift under the limited voltage. As shown in Fig. 7(b), we measured the spectral drift of parallel electrodes (blue) and individual electrodes (red) with respect to the applied voltage. The length of the heating zone is $2 \mathrm{~mm}$. The spectral drift is only 0.3 $\mathrm{nm}$ under the voltage of $20 \mathrm{~V}$ with individual electrodes. When the heating zone is the parallel electrodes design, the 
spectral drift can achieve $4.68 \mathrm{~nm}$ under the voltage of $8 \mathrm{~V}$. Because most voltage sources cannot operate at a very high voltage, the proposed segmented heating method can suitably solve this problem. Fig. 7(c) shows the microscopic image of the contra-directional couplers with parallel electrodes. In addition, the measured reflection spectra with passband of $10 \mathrm{~nm}$ and group delay of $50 \mathrm{ps}$, as Figs. 7(d) and 7(e) shown, maintaining good consistency under different voltages.

a

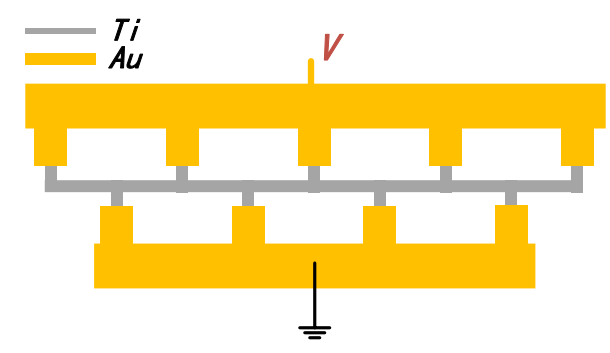

c
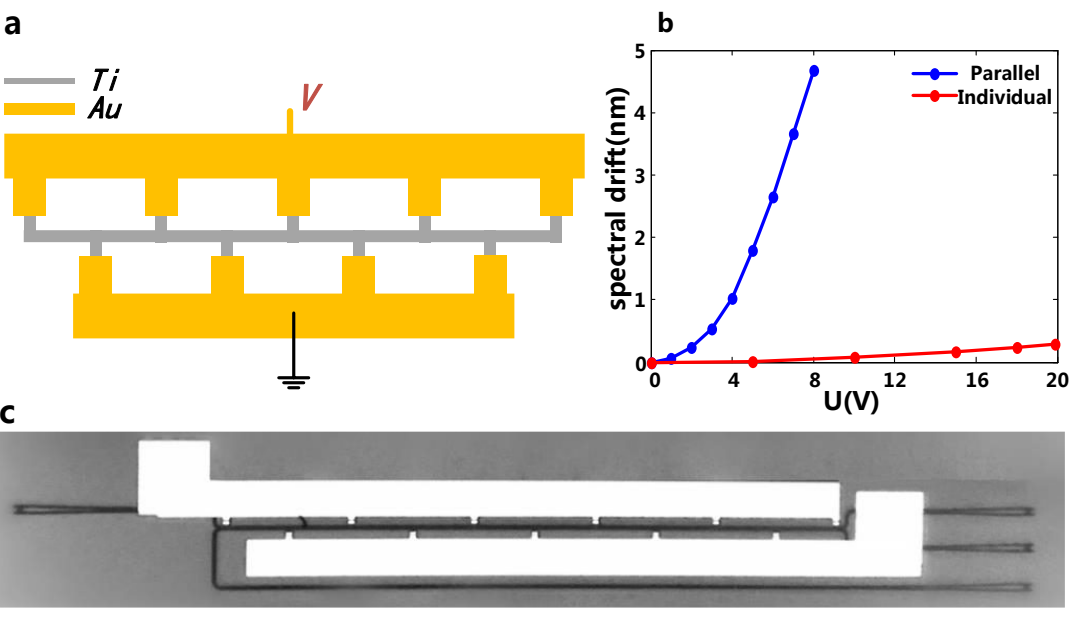

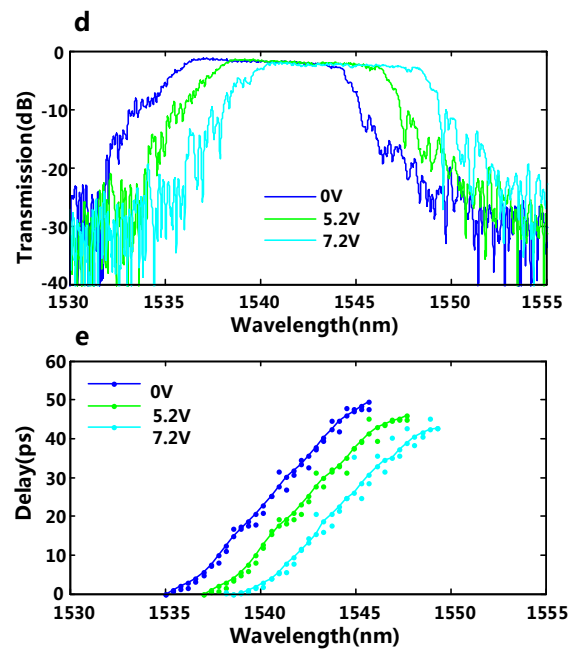

Figure 7. (a) The schematic of the parallel electrodes. (b) Measured spectral drift of parallel electrodes (blue) and individual electrodes (red) with respect to the applied voltage. (c) Microscopic image of the contra-directional couplers with parallel electrodes. (d) Measured reflection spectra under different voltages. (d) Measured group delay under different voltages.

\section{SILICON PHOTONIC MICROWAVE SIGNAL PROCESSOR}

The microwave photonic filter (MPF) has its natural advantages on frequency tunability, electromagnetic immunity, and low energy cost comparing to the traditional electrical microwave filter. For a typical MPF system, the key component is the optical resonator, which determines the MPF performance directly. Recently, a few MPF schemes have been proposed by utilizing silica microsphere [42], silicon nitride resonator [43], silicon microring (MRR) [44] or microdisk resonator (MDR) [45], etc. When it comes to integrated platforms, compared to other platforms such as silicon nitride platform [43] and the indium phosphorus platform [14], silicon platform has its own outstanding superiorities such as low cost, small footprint, good tenability, more suitable for the practical applications. Unfortunately, most MPF schemes based on silicon platform show bad performance of it full width at half maximum (FWHM) with the order of gigahertz. This is because that the silicon resonators normally had the relative low $Q$ factor $\left(10^{4}\right)$ [43-47]. Thus, the improvement of the $\mathrm{Q}$ factor has become an urgent issue.

A narrowband MPF also has variable applications in optoelectronic oscillator and radio frequency (RF) measurements, etc. [48, 49]. Photonic microwave measurement techniques could provide better performance of large bandwidth and anti-disturbance ability than traditional electronic methods. Some integrated schemes which can realize instantaneous frequency measurement (IFM) of unknown frequency $[50,51]$ have been demonstrated, while they are not capable of identifying simultaneous multi-frequencies in complex environments. Furthermore, schemes based on microwave frequency to time mapping have potentials for multi-frequencies measurement.

We fabricated an ultra-high Q MRR on an SOI wafer, the microscope image of MRR is shown in Fig. 8(a), and the measured maximum $\mathrm{Q}$ factor of MRR is $1.14 \times 10^{6}$. The central frequency could be tuned from $2.0 \mathrm{GHz}$ to $18.4 \mathrm{GHz}$, as is shown in Fig. 8(b). We utilize this MRR as the key component to design a narrowband bandpass MPF, whose FWHM is as narrow as $170 \mathrm{MHz}$, the corresponding rejection ratio is $26.5 \mathrm{~dB}$, as Fig. 8 (c) shows. Moreover, we added a microheater on MRR to tune the central frequency of MPF. 

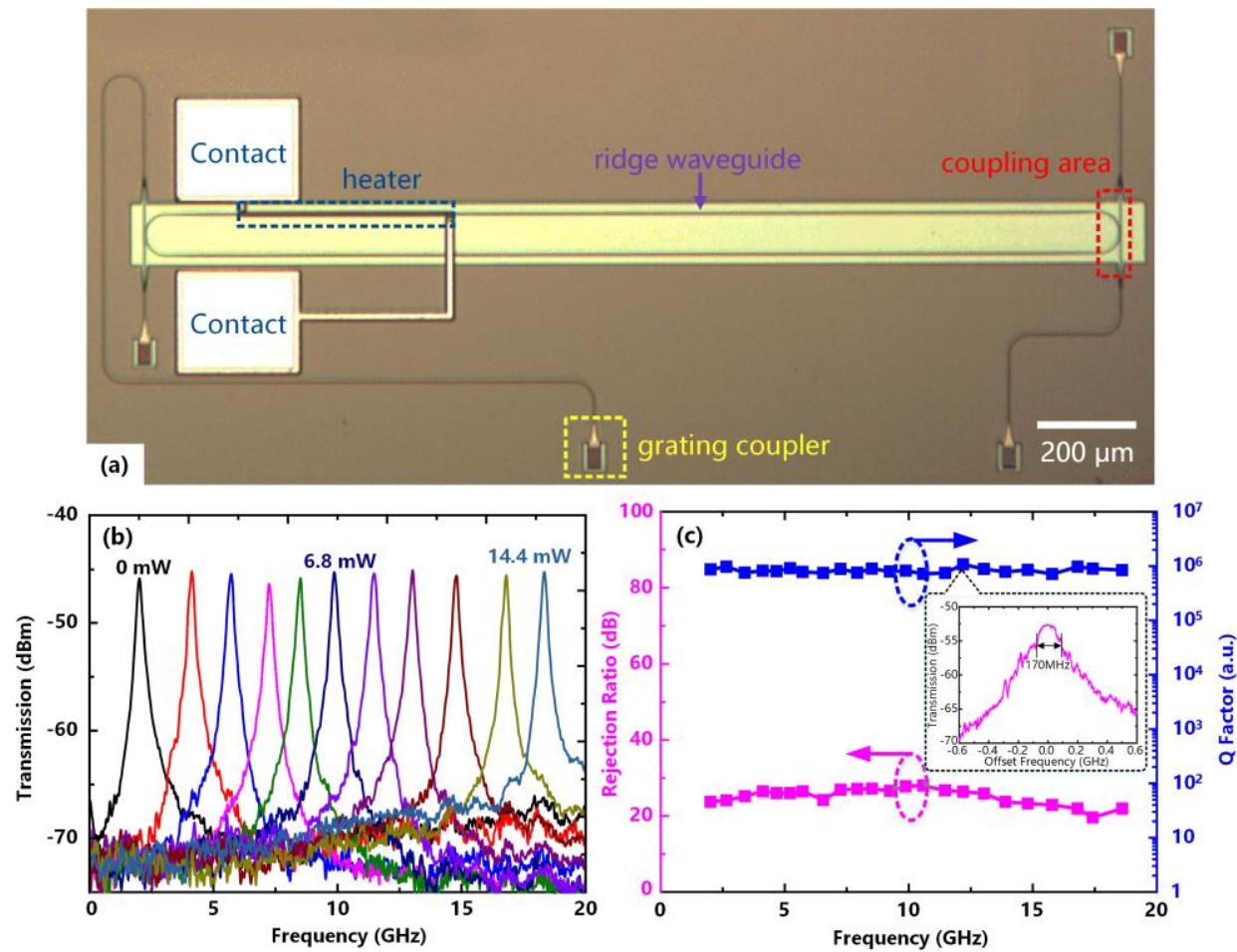

Figure 8. (a) Microscope image of MRR. (b) Tuning range of MPF, from $2.0 \mathrm{GHz}$ to $18.4 \mathrm{GHz}$. (c) Rejection ratio and Q factor of the bandpass MPF.

Moreover, by utilizing the above-mentioned MPF, a scheme of multi-frequency measurement based on frequency-time mapping is proposed. Fig. 9 shows the measured waveforms of different sets of input frequencies. Figs. 9(a) and 9(b) exhibit two-tone frequencies of $2 \mathrm{GHz} \& 2.5 \mathrm{GHz}$ and $27 \mathrm{GHz} \& 28 \mathrm{GHz}$, respectively. Next, a set of 8-tone frequencies from $8 \mathrm{GHz}$ to $11.5 \mathrm{GHz}$, stepped by $0.5 \mathrm{GHz}$ can be separated in the time domain, as shown in Fig 9(c). Finally, 19tone input frequencies from $2 \mathrm{GHz}$ to $20 \mathrm{GHz}$ can be clearly identified as shown in Fig 9(d).
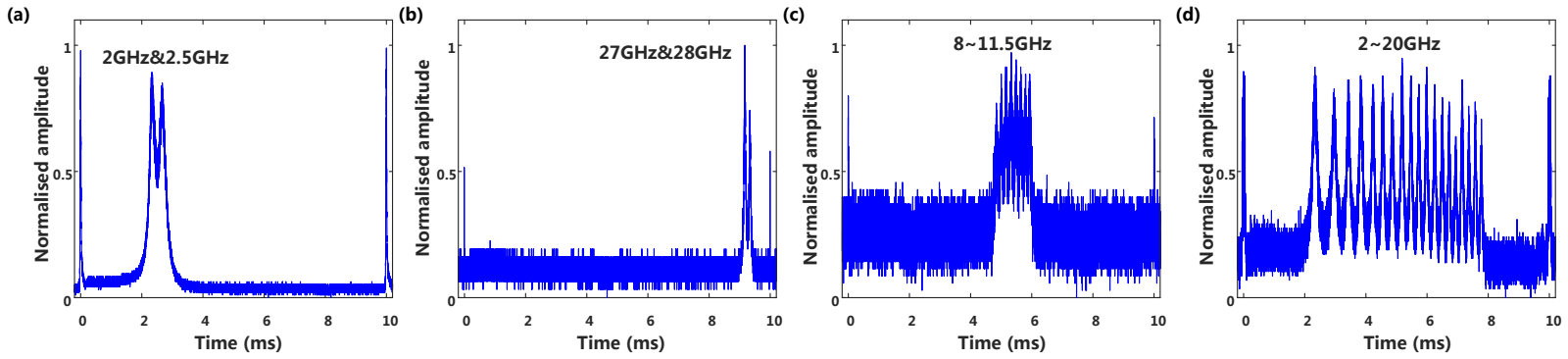

Figure 9. Measured frequency-time mapping waveforms of different set of input frequencies. (a) Two-tone input frequencies of $2 \mathrm{GHz}$ and $2.5 \mathrm{GHz}$. (b) Two-tone input frequencies of $27 \mathrm{GHz}$ and $28 \mathrm{GHz}$. (c) 8-tone input frequencies from $8 \mathrm{GHz}$ to 11.5 GHz. (d) 19-tone input frequencies from $2 \mathrm{GHz}$ to $20 \mathrm{GHz}$.

\section{CONCLUSIONS}

Silicon-based microwave photonics is becoming one of the most important branches of microwave photonics families. In the past decades, fruitful and excellent advances on silicon microwave photonics have emerged due to the mature silicon photonic fabrication foundry. In this paper, we have reviewed some representative progresses on silicon-based integrated microwave photonics in our group, including high efficient microheater on silicon photonic chip, chip scale microwave waveform generator, on-chip true time delay, and microwave photonic processing and measurement. First, 
we have demonstrated an energy-efficient graphene microheater with a tuning efficiency of $1.07 \mathrm{~nm} / \mathrm{mW}$ and power consumption per free spectral range of $3.99 \mathrm{~mW}$. The rise and decay times (10\% to 90\%) are only $750 \mathrm{~ns}$ and $525 \mathrm{~ns}$. The efficient microheater can be widely used in large arrayed microwave processor, such as PAA, switch-based TTD, and so forth. Second, we have demonstrated several typical schemes about microwave arbitrary waveform generation including the finite impulse response synthesis and linearly chirped microwave signal generation. These waveform generators can be widely used in radar system. Third, we have demonstrated an on-chip TTD unit based on chirped grating waveguide. Finally, we have demonstrated some microwave processors, such as MFM and MPF. These chips may have a wide range of applications in silicon-based integrated microwave photonics.

\section{ACKNOWLEDGMENT}

The research is supported under National Natural Science Foundation of China (61622502, and $61475052)$.

\section{REFERENCES}

[1] Jiang, Y., Ma, C., Bai, G., Qi, X., Tang, Y., Jia, Z., Zi, Y., Huang, F. and Wu, T., "Photonic microwave waveforms generation based on time-domain processing," Opt. Express 23(15), 19442-19452 (2015).

[2] McKinney, J.D., Lin, I.S. and Weiner, A.M., "Shaping the power spectrum of ultra-wideband radio-frequency signals," IEEE T Microw Theory 54(12), 4247-4255 (2006).

[3] Fang, X., Wang, D.N. and Li, S., "Fiber Bragg grating for spectral phase optical code-division multiple-access encoding and decoding," JOSA B 20(8), 1603-1610 (2003).

[4] Weiner, A.M., "Ultrafast optical pulse shaping: A tutorial review," Opt. Commun. 284(15), 3669-3692 (2011).

[5] Z. Ao-Ling, D. Jian-Ji, L. Lei, Y. Ting, and Z. Xin-Liang, "Diversity of photonic differentiators based on flexible demodulation of phase signals," Chin. Phys. B 23(3), 033201 (2014).

[6] Kuo, F.M., Shi, J.W., Chiang, H.C., Chuang, H.P., Chiou, H.K., Pan, C.L., Chen, N.W., Tsai, H.J. and Huang, C.B, "Spectral power enhancement in a $100 \mathrm{GHz}$ photonic millimeter-wave generator enabled by spectral line-by-line pulse shaping," IEEE Photon. J. 2(5), 719-727 (2010).

[7] Wang, J., Shen, H., Fan, L., Wu, R., Niu, B., Varghese, L.T., Xuan, Y., Leaird, D.E., Wang, X., Gan, F. and Weiner, A.M., "Reconfigurable radio-frequency arbitrary waveforms synthesized in a silicon photonic chip," Nat. Commun. 6 (2015).

[8] Yan, S., Gao, S., Zhou, F., Ding, Y., Dong, J., Cai, X., and Zhang, X., "Photonic linear chirped microwave signal generation based on the ultra-compact spectral shaper using the slow light effect," Opt. Lett. 42(17), 3299-3302 (2017).

[9] Liao, S., Ding, Y., Dong, J., Yan, S., Wang, X., and Zhang, X., "Photonic arbitrary waveform generator based on Taylor synthesis method," Opt. Express 24(21), 24390-24400 (2016).

[10] Zhang, W. and Yao, J., "Silicon-Based Integrated Microwave Photonics," J. Quantum Electronics 34(1):1-7 (2016).

[11] Zhang, W., Ehteshami, N., Liu, W. and Yao, J., "Silicon-based on-chip electrically tunable sidewall Bragg grating Fabry Perot filter," Opt. Lett. 40(13):3153-3156 (2015).

[12] Rasras, M.S., Madsen, C.K., Cappuzzo, M.A., Chen, E., Gomez, L.T., Laskowski, E.J., Griffin, A., Wong-Foy, A., Gasparyan, A., Kasper, A. and Le Grange, J., "Integrated resonance-enhanced variable optical delay lines," IEEE Photon. Technol. Lett. 17(4), 834-836 (2005).

[13] Pérez, D., Gasulla, I., Crudgington, L., Thomson, D.J., Khokhar, A.Z., Li, K., Cao, W., Mashanovich, G.Z. and Capmany, J., "Multipurpose silicon photonics signal processor core," Nat. Commun. 8(1), 636 (2017).

[14] Fandiño, J.S., Muñoz, P., Doménech, D. and Capmany, J., "A monolithic integrated photonic microwave filter," Nat. Photon. 11(2), 124-129 (2017).

[15] Pagani, M., Vu, K., Choi, D.Y., Madden, S.J., Eggleton, B.J. and Marpaung, D., "Instantaneous microwave frequency measurement using four-wave mixing in a chalcogenide chip," Opt. Commun. 373,100-104 (2017).

[16] Jiang, H., Marpaung, D., Pagani, M., Vu, K., Choi, D.Y., Madden, S.J., Yan, L. and Eggleton, B.J., "Wide-range, high-precision multiple microwave frequency measurement using a chip-based photonic Brillouin filter," Optica 3(1), 30-34 (2016). 
[17] Marpaung, D., Morrison, B., Pagani, M., Pant, R., Choi, D.Y., Luther-Davies, B., Madden, S.J. and Eggleton, B.J., "Low-power, chip-based stimulated Brillouin scattering microwave photonic filter with ultrahigh selectivity," Optica 2(2), 76-83 (2015).

[18] Capmany, J., Gasulla, I. and Pérez, D., "Microwave photonics: The programmable processor," Nat. Photon. 10(1), 6-8 (2016).

[19] Dong, P., Qian, W., Liang, H., Shafiiha, R., Feng, D., Li, G., Cunningham, J.E., Krishnamoorthy, A.V. and Asghari, M., "Thermally tunable silicon racetrack resonators with ultralow tuning power," Opt. Express 18(19), 20298-20304 (2010).

[20] Sun, P., and Reano, R. M. "Submilliwatt thermo-optic switches using free-standing silicon-on-insulator strip waveguides," Opt. Express, 18(8), 8406-8411 (2010).

[21] Li, X., Xu, H., Xiao, X., Li, Z., Yu, Y., and Yu, J. "Fast and efficient silicon thermo-optic switching based on reverse breakdown of pn junction," Opt. Lett., 39(4), 751-753 (2014).

[22] Yu, L., Yin, Y., Shi, Y., Dai, D., and He, S. "Thermally tunable silicon photonic microdisk resonator with transparent graphene nanoheaters," Optica, 3(2), 159-166 (2016).

[23] Yan, S., Zhu, X., Frandsen, L.H., Xiao, S., Mortensen, N.A., Dong, J. and Ding, Y., "Slow-light-enhanced energy efficiency for graphene microheaters on silicon photonic crystal waveguides," Nature Communications, 8 (2017).

[24] Fontaine, N.K., Scott, R.P. and Yoo, S.J.B., "Dynamic optical arbitrary waveform generation and detection in InP photonic integrated circuits for Tb/s optical communications," Opt. Commun. 284(15), 3693-3705 (2011).

[25] Roos, P.A., Reibel, R.R., Berg, T., Kaylor, B., Barber, Z.W. and Babbitt, W.R., "Ultrabroadband optical chirp linearization for precision metrology applications," Opt. Lett. 34(23), 3692-3694 (2009).

[26] Li, X., Dong, J., Yu, Y. and Zhang, X., "A Tunable Microwave Photonic Filter Based on an All-Optical Differentiator," IEEE Photon. Technol. Lett. 23(5), 308-310 (2011).

[27] Ferrera, M., Park, Y., Razzari, L., Little, B.E., Chu, S.T., Morandotti, R., Moss, D.J. and Azaña, J., "On-chip CMOS-compatible all-optical integrator," Nat. Commun. 1(3), 29 (2010).

[28] Ashrafi, R., Dizaji, M.R., Cortés, L.R., Zhang, J., Yao, J., Azaña, J. and Chen, L.R., "Time-delay to intensity mapping based on a second-order optical integrator: application to optical arbitrary waveform generation," Opt. Express 23, 16209-16223 (2015).

[29] Liao, S., Ding, Y., Dong, J., Yang, T., Chen, X., Gao, D. and Zhang, X., "Arbitrary waveform generator and differentiator employing an integrated optical pulse shaper," Opt. Express 23(9), 12161-12173 (2015).

[30] Liao, S., Ding, Y., Peucheret, C., Yang, T., Dong, J. and Zhang, X., "Integrated programmable photonic filter on the silicon-on-insulator platform," Opt. Express 22(26), 31993-31998 (2014).

[31] Liao, S., Ding, Y., Dong, J., Wang, X. and Zhang, X., "125 GHz microwave signal generation employing an integrated pulse shaper," J Lightwave Technol. 35(13), 2741-2745 (2017).

[32] Doerr, C.R., Chandrasekhar, S., Winzer, P.J., Chraplyvy, A.R., Gnauck, A.H., Stulz, L.W., Pafchek, R., Burrows, E., "Simple Multichannel Optical Equalizer Mitigating Intersymbol Interference for 40-Gb/s Nonreturn-To-Zero Signals," J Lightwave Technol. 22(1), 249-256 (2004).

[33] Corral, J.L., Marti, J., Fuster, J.M. and Laming, R.I., "True time-delay scheme for feeding optically controlled phased-array antennas using chirped-fiber gratings," IEEE Photon. Technol. Lett. 9(11), 1529-1531 (1997).

[34] Zhang, W., Yao, J., "Photonic Generation of Linearly Chirped Microwave Waveforms Using a Silicon-Based OnChip Spectral Shaper Incorporating Two Linearly Chirped Waveguide Bragg Gratings," J. Lightwave Technol. 33, 5047-5054 (2015).

[35] Xie, J., Zhou, L., Li, Z., Wang, J., Chen, J., "Seven-bit reconfigurable optical true time delay line based on silicon integration," Opt. Express 22(19), 22707-22715 (2014).

[36] Xie, J., Zhou, L., Zou, Z., Wang, J., Li, X., Chen, J., "Continuously tunable reflective-type optical delay lines using microring resonators," Opt. Express 22(1), 817-823 (2014).

[37] Melloni, A., Morichetti, F., Ferrari, C., Martinelli, M., "Continuously tunable 1 byte delay in coupled-resonator optical waveguides," Opt. Lett. 33(20), 2389-2391 (2008).

[38] Morichetti, F., Melloni, A., Breda, A., Canciamilla, A., Ferrari, C., Martinelli, M., "A reconfigurable architecture for continuously variable optical slow-wave delay lines," Opt. Express 15(25), 17273-17282 (2007).

[39] Giuntoni, I., Stolarek, D., Kroushkov, D.I., Bruns, J., Zimmermann, L., Tillack, B., Petermann, K., "Continuously tunable delay line based on SOI tapered Bragg gratings," Opt. Express 20(10), 11241-11246 (2012).

[40] Shi, W., Veerasubramanian, V., Patel, D., Plant, D.V., "Tunable nanophotonic delay lines using linearly chirped contradirectional couplers with uniform Bragg gratings," Opt. Lett. 39(3), 701-703 (2014). 
[41] Wang, X., Liao, S., and Dong, J., "Optical true time delay based on contradirectional couplers with single sidewallmodulated Bragg gratings," Proceedings of the SPIE, Volume 10026, 6 (2016).

[42] Liu, Y., Yu, Y., Yuan, S., Xu, X. and Zhang, X., "Tunable megahertz bandwidth microwave photonic notch filter based on a silica microsphere cavity," Opt. Lett. 41(21), 5078-5081 (2016).

[43] Liu, Y., Hotten, J., Choudhary, A., Eggleton, B.J. and Marpaung, D., "All-optimized integrated RF photonic notch filter," Opt. Lett. 42(22), 4631-4634 (2017).

[44] Dong, J., Liu, L., Gao, D., Yu, Y., Zheng, A., Yang, T. and Zhang, X., "Compact notch microwave photonic filters using on-chip integrated microring resonators," IEEE Photon. J. 5(2), 5500307-5500307 (2013).

[45] Zhang, W. and Yao, J., "Silicon-Based Single-Mode On-Chip Ultracompact Microdisk Resonators With Standard Silicon Photonics Foundry Process," J. Lightwave Technol. 35(20), 4418-4424 (2017).

[46] Liu, L., Yang, Y., Li, Z., Jin, X., Mo, W. and Liu, X., "Low power consumption and continuously tunable alloptical microwave filter based on an opto-mechanical microring resonator," Opt. Express 25(2), 960-971 (2017).

[47] Capmany, J. and Novak, D., "Microwave photonics combines two worlds," Nat. Photon. 1(6), 319-330 (2007).

[48] Capmany, J., Ortega, B. and Pastor, D., "A tutorial on microwave photonic filters," J. Lightwave Technol. 24(1), 201-229 (2006).

[49] Burla, M., Wang, X., Li, M., Chrostowski, L. and Azaña, J., "Wideband dynamic microwave frequency identification system using a low-power ultracompact silicon photonic chip," Nat. Commun. 7, 13004 (2016).

[50] Jiang, H., Marpaung, D., Pagani, M., Vu, K., Choi, D.Y., Madden, S.J., Yan, L. and Eggleton, B.J., "Wide-range, high-precision multiple microwave frequency measurement using a chip-based photonic Brillouin filter," Optica 3(1), 30-34 (2016).

[51] Zou, X., Lu, B., Pan, W., Yan, L., Stöhr, A. and Yao, J., "Photonics for microwave measurements," Laser \& Photonics Reviews 10, 711-734 (2016). 